\title{
Arrays of annular cryogenic antennas with SINIS bolometers and cryogenic receivers for SubTHz observatories
}

\author{
M. Tarasov ${ }^{1}$, A. Gunbina ${ }^{2,5}$, M.Mansfeld $^{2,5}$, G. Yakopov ${ }^{4}$, A.Chekushkin ${ }^{1}$, R. Yusupov $^{1}$, \\ S.Lemzyakov ${ }^{6}$, V. Edelman ${ }^{6}$, and V. Vdovin ${ }^{2,3,5}$, \\ ${ }^{1}$ V.Kotelnikov Institute of Radio Engineering an d Electronics RAS \\ ${ }^{2}$ Institute of Applied Physics RAS \\ ${ }^{3}$ P.N. Lebedev Physical Institute of the Russian Academy of Sciences \\ ${ }^{4}$ Special Astrophysical Observatory RAS \\ ${ }^{5}$ Nizhny Novgorod State Technical University n.a. R.E. Alekseev \\ ${ }^{6}$ P.Kapitza Institute for Physical Problems RAS
}

Series and parallel arrays of annular antennas optimized for $350 \mathrm{GHz}$ frequency band with SINIS (Superconductor - Insulator - Normal metal - Insulator-Superconductor) bolometers and cryogenically cooled receivers for series of modern SubTHz observatories have been designed, fabricated and studied. The detectors are matched to incoming beam by an immersion sapphire lens. In the low background power case a voltage responsivity approaches $1.5^{*} 10^{9} \mathrm{~V} / \mathrm{W}$ corresponding to $\mathrm{NEP}=10^{-17} \mathrm{~W} / \mathrm{Hz}^{1 / 2}$. The current responsivity for parallel array is $4 * 10^{4} \mathrm{~A} / \mathrm{W}$ and shot noise limited intrinsic noise equivalent power is $\mathrm{NEP}=4 * 10^{-18} \mathrm{~W} / \mathrm{Hz}^{1 / 2}$. At the background radiation temperature level of $2.7 \mathrm{~K}$ the responsivity to variations of radiation temperature is over $75 \mu \mathrm{V} / \mathrm{K}$. The intrinsic time constant of the bolometer array is $0.1-0.2 \mu \mathrm{s}$.

\section{Introduction}

Observation of the CMB radiation anisotropy and some other objects of cold Universe are the priority tasks for ambitious projects in radioastronomy where some new observatories are under development: international space observatory Millimetron, international observatory Suffa (Uzbekistan) and QTT observatory, China. Technical requirements for receivers of listed observatories are rather contradictive, high sensitivity should be combined with a wide dynamic range and a low time constant. Roomtemperature semiconducting electronics practically expired potential for improvement and superconducting devices offer promising potential. Only superconductors (TES, KID and other) and cryogenically cooled electronics gives possibility to provide new observatories by proper instruments. A realistic alternative to transition edge sensors (TES) can be SINIS bolometers that provide high responsivity, wide dynamic range and high speed. In present contribution we study performance of such bolometers integrated in series and parallel arrays.

\section{Design and fabrication}

Bolometers were integrated in annular ring antennas designed for central frequency of $345 \mathrm{GHz}$. Antennas were arranged in series and parallel arrays to increase saturation power and dynamic range. The layout of SINIS bolometers was modified to increase the volume of superconducting electrodes up to 100 times (area from $2 \mu \mathrm{m}^{2}$ in our previous designs [1, 2] to $250 \mu^{2}$ ) to reduce overheating by strong dilution of hot quasiparticles in such big volume. Another layout modification is in eliminating of additional layer of so-called thin gold that suppressed superconductivity and connecting $\mathrm{Al}$ electrodes directly to thick TiAuPd antennas that also improve cooling of superconducting electrodes.

\section{Measurement setup}

Our previous arrangement of experiment was based on a back-to-back horn that reduces a role of antenna dimensions on overall spectral characteristics and beampattern. In present research we have developed results of previous results $[1,2]$ and used immersion sapphire lens instead of horn. Such substrate lens provides direct illumination of array, avoid substrate modes, and increase the gain of planar antenna. Top view of dilution cryostat [3] with 3 such lenses is presented in Fig. 1. Each sample was connected to room-temperature electronics through cold resistors that provide suppression of interferences and noise. The sapphire hemisphere lens diameter is $8 \mathrm{~mm}$. Total extension for hyperhemisphere should be $1.5 \mathrm{~mm}$, in this case antenna on the chip $0.28 \mathrm{~mm}$ thick is placed in the second focus of elliptical lens. Such elliptical lens convert a spherical wave into a planar wave.

Cryogenic fast radiation source comprising $\mathrm{NiCr}$ film on sapphire substrate was equipped with a thermometer and placed on $0.5 \mathrm{~K}$ temperature stage. Bandpass and lowpass filters between such source and sample determine the bandwidth of irradiating power.
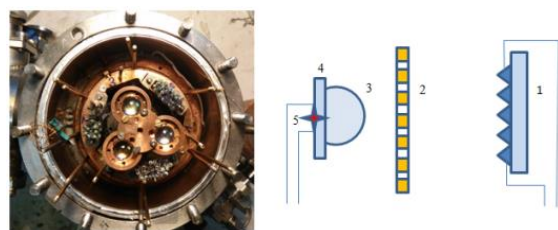

Fig. 1. Photo of opened cryostat with three samples on three sapphire lenses (left), and schematics of sensitivity measurements (right): 1 fast radiation source, 2 bandpass and lowpass filters, 3 hemisphere lens, 4 extension substrate, 5bolometer array

\section{Experimental results}

The asymptotic resistance for single ring is $1700 \Omega$, for series array of 25 rings it is $39 \mathrm{\kappa} \Omega$, and for 25 parallel rings it is $65 \Omega$. Responsivity of bolometers was measured by varying the radiation source temperature from initial $0.5 \mathrm{~K}$ by applying DC 
current through $\mathrm{NiCr}$ film on sapphire substrate suspended on thin wires. We compared voltage response of series array for variation of bath temperature and for variation of radiation temperature (see Fig. 2). Dependencies are identic that is a clear proof of thermal mechanism of response contrary to our results with previous generation of samples with lower volume of superconducting electrodes and different geometry of antennas. Position of maximum is slightly shifted from about $1 \mathrm{nA}$ at low radiation power up to about $1.6 \mathrm{nA}$ for higher power. Estimated power level calculated for receiving of single mode.

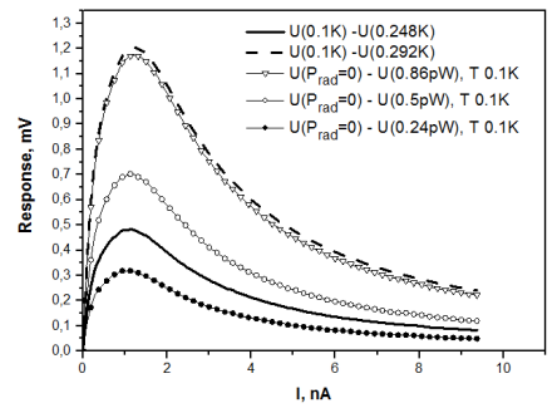

Fig. 2. Voltage response for temperature $(0.248 \mathrm{~K}$ and $0.292 \mathrm{~K}$ ) and radiation power

Illustration for saturation and dynamic range is presented in Fig. 3 for series array at bias currents $1 \mathrm{nA}$ and $1.6 \mathrm{nA}$ and for single ring response voltage multiplied by factor of 25 for comparison. Both dependencies can be fitted by a simple relation presented as a line. For radiation power of $2 \mathrm{pW}$ and bias current $1.6 \mathrm{nA}$ the responsivity $\mathrm{dV} / \mathrm{dP}$ is reduced only by $30 \%$ compared to low radiation power case. Small reducing of response for single ring can be due to its position at the edge of chip with lower illumination power. We also compared response of series array for the same signal on the bath temperature (Fig. 4). Three times increase of temperature from 0.1 to $0.3 \mathrm{~K}$ leads to decrease of maximum response only by a factor of 1.5. A figure of merit for astronomic applications is radiation temperature responsivity (Fig. 5). At the background power level of $2.7 \mathrm{~K}$ the responsivity to variations of radiation temperature is over $75 \mu \mathrm{V} / \mathrm{K}$. For amplifier noise limited case of $7 \mathrm{nV} / \mathrm{Hz}^{1 / 2}$, the detection of relative radiation temperature variation is at the level of $4 * 10^{-5}$.

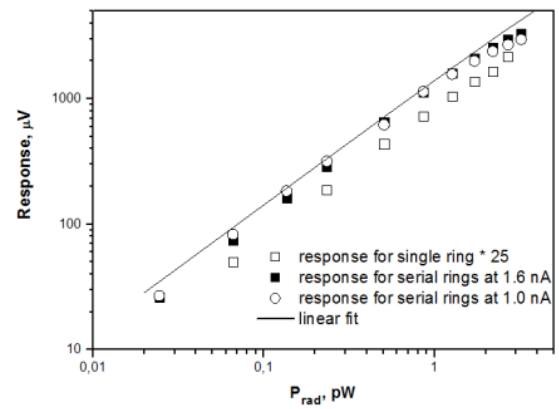

Fig. 3. Response at $0.1 \mathrm{~K}$ for series array of 24 rings, single ring $\mathrm{x} 25$, and linear fitting

The time constant of such detector (Fig. 6) have been measured by response for the heating pulses of $85 \mathrm{~V}$ amplitude and duration of $1 \mu \mathrm{s}$. Fitting by exponent of signal increase after the end of heating pulse shows that the overall time constant of receiver is $\tau_{\text {sum }} \approx 0.55 \mu \mathrm{s}$. The main impact to this time constant is due to the readout amplifier that is $\tau_{\mathrm{amp}}$ $\approx 0.4 \mu$ s measured separately with test signal from optocoupler pair. From these data we can estimate the intrinsic time constant of the bolometer as 0.1-0.2 $\mathrm{s}$.

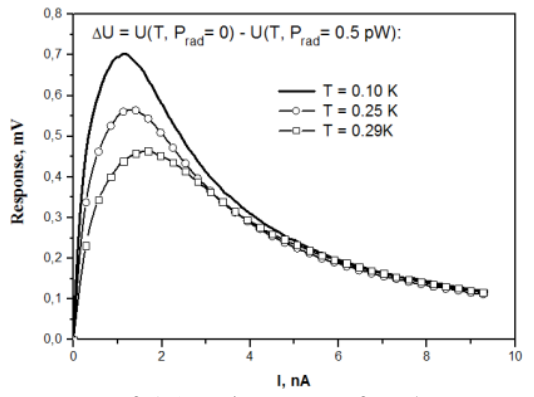

Fig. 4. Response of 25 series array for the same radiation power of $0.5 \mathrm{pW}$ measured at bath temperatures $0.1 \mathrm{~K}, 0.25$ $\mathrm{K}$, and $0.29 \mathrm{~K}$

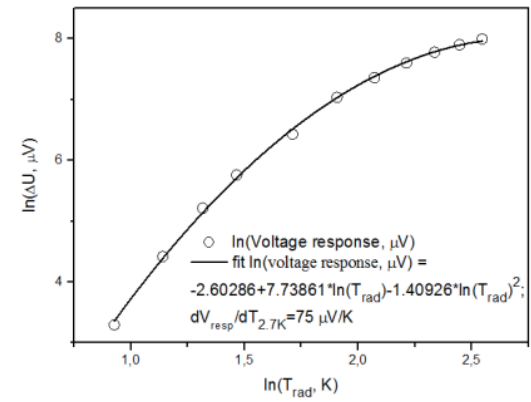

Fig. 5. Logarithmic plot of voltage response for series array at $0.1 \mathrm{~K}$. Radiation temperature responsivity is $75 \mu \mathrm{V} / \mathrm{K}$ at background temperature of $2.7 \mathrm{~K}$

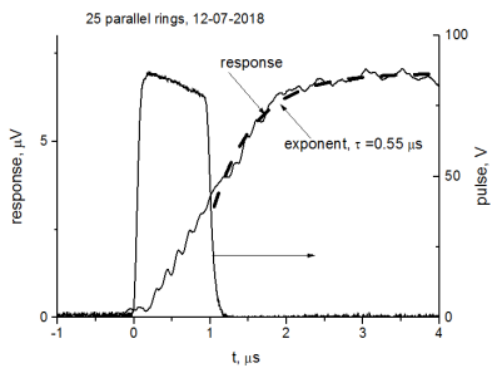

Fig. 6. Probing pulse applied to radiation source (right axis), and output voltage response (left axis) together with fitting by exponent (dashed)

\section{References}

1. S. Mahashabde, A. Sobolev, A. Bengtsson, et al. A frequency selective surface based focal plane receiver for the OLIMPO balloon-borne telescope //IEEE Trans. on Terahertz Science and Technology. 2015.V.5,N1. P.145.

2. S. Mahashabde, A. Sobolev, M. Tarasov, et al. Planar frequency selective bolometric array at $350 \mathrm{GHz} / / \mathrm{IEEE}$ Trans. on TST. 2015.V.5, No 1. P. 37-43.

3. V.S. Edelman. A dilution microcryostat-insert //Instrum. and Exp. Tech. 2009. V. 52, No 2. P. 301-307.

4. G.M. Rebeiz. Millimeter-wave and Terahertz integrated circuit antennas // Proc. of the IEEE. 1992. V. 80, No 11, P. 1748-1770

\section{Acknowledgements}

The work was supported by the IAP RAS state program (project No. 0035-2014-0021) and by the Basic Research Program of the Presidium of RAS No. 32 Project "PRIZE" 